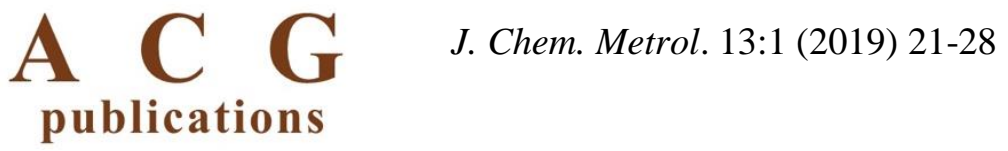

journal of chemical metrology

\title{
Investigation of caffeine concentrations in sport supplements and inconsistencies in product labelling
}

\section{Eda Okuroglu $\oplus^{1}$, Tugba Tekin $\oplus^{1}$, Merve Kuloglu $\oplus^{1}$, Selda Mercan $\odot 1$, Isil Bavunoglu $\oplus^{2}$, Munevver Acikkol ${ }^{\oplus}{ }^{1}$ and Zeynep Turkmen ${ }^{(1 *}$}

\author{
${ }^{1}$ Department of Science, Institute of Forensic Sciences, Istanbul University-Cerrahpasa, 34500, \\ Buyukcekmece, Istanbul, Türkiye \\ ${ }^{2}$ Department of Internal Medicine, Cerrahpasa Medical Faculty, Istanbul University-Cerrahpasa \\ 34098, Cerrahpasa, Istanbul, Türkiye
}

(Received April, 17, 2019; Revised June 13, 2019; Accepted June 14, 2019 )

\begin{abstract}
Caffeine is a substance that can be easily consumed in daily nutrition as well as in sport supplements. Exceeding the daily-recommended dose for caffeine may cause serious health problems. Studies illustrated that many concerns have been raising about the incompatibility between labels on sport supplements and their contents. The present study aimed to investigate caffeine contents in sport supplements purchased from Turkey market for the first time and to evaluate label information on products. Caffeine concentrations were determined in 41 sport supplements and possible adverse effects were emphasized considering calculated daily consumption doses. Seventeen samples among 41 products were found to be caffeine containing, ranging from 4.52 to $471 \mathrm{mg} / \mathrm{mL}$. Three of caffeine containing products were undeclared their caffeine ingredients. Although three products were not specified caffeine their amounts, findings were found above the daily-recommended caffeine limit (400 $\mathrm{mg}$ /day). Significant differences were observed between the quantities indicated on the labels and the determined caffeine amounts in 7 products $(\mathrm{p}<0.05)$. Eight of 17 caffeine labelled products were found between 151-948\% higher than stated label information. Overall, $29.4 \%$ of caffeine containing products $(\mathrm{n}=17)$ were found over the recommended intake. Consuming more than the daily-recommended dose may cause severe adverse reactions and consumers may experience serious health risks such as arterial hypertension, coronary heart disease, as well as sleep problems, irritability, anxiety, headache, and restlessness. Considering these health risks, awareness of consumers and accurate product labels on sport supplements gain more importance to prevent people from undesired consequences.
\end{abstract}

Keywords: Sport supplement; caffeine; GC-MS; label inconsistency; daily intake. @ 2019 ACG Publications. All rights reserved.

\section{Introduction}

Caffeine (1, 3, 7-trimethylxanthine), was discovered by German chemist Friedich Ferdinand Runge in 1819 as an alkaloid and is known by the name of matein or guaranin. In nature, it can be found in a number of known crops such as guarana, cola nuts, cocoa and tropical plant species such as coffee, black tea and Paraguay [1,2]. Caffeine is commonly involved in sport supplements, which are used to

\footnotetext{
*Corresponding author: E-Mail: zeytur@gmail.com
} 
enhance sport performance. The presence of caffeine in these products has various effects in the body. As a stimulant, caffeine increases the secretion of cortisol hormones in the body and the amount of adrenalin in the blood. With the release of adrenaline, the heart rate, the number of breathing and the free fatty acid levels increase in the blood, as well as fat burning and energy production [3]. Caffeine is rapidly absorbed from the gastrointestinal tract and reaches peak concentration in 1-2 hour through liver circulations and metabolizes into paraxanthine, theophylline and xanthine metabolites $[3,4]$. The most important mechanism of action is the binding of adenosine receptors to its antagonists $[4,5]$. Another known mechanism of action for caffeine is to increase the beta-endorphin level in plasma, which leads reduction in pain during activity and improvement in resistance to performance [6,7].

Caffeine consumption increases the endurance capacity during workout by allowing to exercise for a longer time [8,9]. Due to its lipophilic character, it easily passes to the blood-brain barrier and acts on the central nervous system [7]. That is the reason why, many pre-training supplements contain caffeine as a stimulant compound. According to Food and Drug Administration (FDA) and European Food Safety Authority (EFSA), the effective dose of caffeine is 3-6 mg/kg, which corresponds to 400 $\mathrm{mg} /$ day for a $70 \mathrm{~kg}$ adult. The idea is to maintain a limit for the daily caffeine consumption to moderate amounts for a healthy society $[10,11]$. Additionally, a high tolerance is seen for the use of caffeine in the World of professional sports. For example, the World Anti-Doping Committee has not included caffeine in the prohibited substance list [12].

However, the International Olympic Committee has assigned $12 \mu \mathrm{g} / \mathrm{mL}$ concentration for caffeine in urine as an acceptable limit. This corresponds to a range about 3-6 mg/kg (low-to-medium doses) [13]. High doses are indicated as $\geq 9 \mathrm{mg} / \mathrm{kg}$ and the possible side effects are reported as increased heart rate, excessive sweating, tremor and vomiting [14]. On the other hand, certain populations / vulnerable groups, such as adolescents, pregnant or nursing women, and elderly or hypertensive individuals may experience side effects even at lower caffeine doses.

For aforementioned reasons, it is recommended that healthy pregnant and nursing women should not consume more than $300 \mathrm{mg}$ of caffeine per day. Additionally, adolescents should not take over $2.5 \mathrm{mg} / \mathrm{kg}$-day [15]. The danger is that caffeine-containing substances do not accelerate fat burning solely; they may also cause serious side effects due to incorrect dosages and non-conforming labeling [16].

In other respects, since caffeine is a stimulant, which can be easily consumed in daily nutrition (coffee, chocolate, tea etc.), use of sport supplements has a high potential to reveal adverse effects even in optimum usage. That is why; the daily safe limit of caffeine can be easily exceeded by consuming caffeine containing sport supplements and beverages in the same day. Studies illustrated that many concerns have been raising about the incompatibility between labels on the products and their contents. Many of the manufacturers of these products do not want to disclose the exact amounts of active ingredients in their particular blends to hide their formulations [16]. However, an increase or misuse in caffeine intake may lead to acute dehydration, which will cause disturbance in fluid-electrolyte balance and thermoregulation. In this case, negative consequences will be inevitable on both performance and vital signs [14].

A study reported by Bessada et al. was conducted regarding to product's label claims and recommended daily intakes. In this research, it was found that approximately $11 \%$ of the examined products were at or above the daily intake limit $(400 \mathrm{mg})$ recommended by EFSA and FDA. In addition, they suggested to analyze caffeine existence in products and to compare those with the labelled content [16]. Numerous analytical studies have also been conducted to determine the caffeine concentration in human biological matrices, supplementary foods and/or beverages so far, with high performance thin layer chromatography, high performance liquid chromatography, liquid chromatography-mass spectrometry and gas chromatography-mass spectrometry (GC-MS) [1,17-21].

However according to our knowledge, there is no study in the literature determining the caffeine content in sport supplements available in Turkey market using aforementioned analytical techniques. The aim of this presented study was to investigate the caffeine contents and incompatibilities on labelling in sport supplements for the first time in Turkey market. In addition, clinical evaluation of those determined concentrations was presented by considering daily caffeine intake limits. 


\section{Experimental}

\subsection{Chemicals and Equipments}

The caffeine (CAF) standard (99.6\% purity) and n-docosane as an internal standard (IS) were purchased from Sigma (St. Louis, MO, USA). Methanol and dichloromethane were purchased from Merck (99,8-100\%, Darmstadt, Germany). All the solvents used were of LC grade. A Direct-Q3 ${ }^{\text {TM }}$ water system (Millipore, Bedford, MA, USA) was used in the laboratory to produce deionized water. Sodium hydroxide, ethyl acetate, n-heptane and hydrochloric acid (37\%) were purchased from Sigma Aldrich (St. Louis, Missouri, ABD).

The experiments were conducted with nitrogen and argon gases $(>99.999 \%$ purity, Okser, Turkey). Evaporation under nitrogen stream was conducted with a HyperVap HV-300 from Gyrozen (Daejeon, Rep. of Korea) during sample preparation. A Direct-Q UV 3 ultrapure water system was acquired from Millipore (Molsheim, France) $(18.2 \mathrm{M} \Omega \mathrm{cm}$ ). Nitrogen was generated via PEAK Scientific Genius 1051 (Glasgow, Scotland).

\subsection{Instrumental Analysis}

Agilent HP 7820A Gas Chromatography device and 5977E Mass Spectrometer (USA), equipped with a $30 \mathrm{~m} \times 0.25 \mathrm{~mm}$ fused silica capillary column (HP-5MS 5\% Phenyl Methyl Siloxane, film thickness $0.25 \mu \mathrm{m}$ - Agilent Part No. 19091S-433) were used to perform analytical measurements. The data was collected on a Chemstation software (G1701FA) for data acquisition and processing. Chromatographic separation was achieved on a HP-5MS (30 m x $25 \mathrm{~mm}$ (i.d.) $\times 25 \mu \mathrm{m}$ film thickness). The oven temperature gradient was start at $110^{\circ} \mathrm{C}$, held for $1 \mathrm{~min}$, ramped to $250^{\circ} \mathrm{C}$ at $15^{\circ} \mathrm{C} / \mathrm{min}$ where it was held for $12 \mathrm{~min}$. Total run time was $22.33 \mathrm{~min}$. The temperatures of the injection port and interface were set at $250^{\circ} \mathrm{C}$ and $230^{\circ} \mathrm{C}$, respectively. One $\mu \mathrm{L}$ volume of each sample was injected in split mode at a split ratio of 5:1 to minimize the matrix effect. Helium was used as a carrier gas at a final flow rate of $0.9 \mathrm{~mL} / \mathrm{min}$. The inlet and the transfer line temperatures were both set at $290^{\circ} \mathrm{C}$. Ion source temperature was set at $250^{\circ} \mathrm{C}$. Acquisition delay was set at 120 seconds and the mass spectrometer set to scan from 50 to 550 mass units with mass spectra collected at 10 spectra/ second. The GC-MS method of this study was conducted in selected ion monitoring (SIM) mode. Ions used for identification were $\mathrm{m} / \mathrm{z}$ 67, 109, $194 \mathrm{~m} / \mathrm{z}$ and 57, 71, $310 \mathrm{~m} / \mathrm{z}$ for n-docosane (IS). Mass spectrum of CAF and IS were shown in the supporting information (Figure S1 and S2).

\subsection{Sample Collection and Preparation}

For this study, 41 sport supplements in powder and tablet forms were supplied from nutrition markets and fitness centers in Turkey. Types of products investigated in this study were; performance enhancer $(n=11)$, amino acid $(n=5)$, amino acids - glutamine mix $(n=2)$, L-carnitine $(n=1)$, amino acid - arginine $(\mathrm{n}=1)$, amino acid - creatine $(\mathrm{n}=1)$, branched chain amino acids (BCAA) ( $\mathrm{n}=6)$, carbohydrate $(n=3)$, protein $(n=2)$, and whey protein $(n=9)$. Among these 41 products, 14 of them were labelled for the presence of caffeine content, while 11 of these labelled products were stated the caffeine amount in each serving size.

Tablet forms were grind into a fine powder using a mortar to obtain homogeneity. Ten $\mathrm{mg}$ of each sample was dissolved in $5 \mathrm{~mL}$ distilled water and vortexed up to complete solubility. Sodium hydroxide $(1 \mathrm{~mL}-0.1 \mathrm{M})$ was added to $100 \mu \mathrm{L}$ aliquot of the aqueous solution. After incubation at $45^{\circ} \mathrm{C}$ for 15 min, $\mathrm{pH}$ was adjusted to 3 with $0.1 \mathrm{M}$ hydrochloric acid. The liquid-liquid extraction process was performed in triplicates using $5 \mathrm{~mL}$ ethyl acetate:n-heptane solvent mixture (1:1) and all organic phases were pooled for evaporation to dryness under nitrogen at $45^{\circ} \mathrm{C}$. Finally, samples were reconstructed with $1 \mathrm{~mL}$ methanol including $100 \mu \mathrm{g} / \mathrm{mL}$ IS and analyzed by GC-MS instrument. 


\subsection{Method Validation}

Retention time and selected ions of compounds (CAF and IS) were determined by injecting reference standard solutions. Both validation studies and supplement samples were injected in three replicates during the study. To avoid contamination or carryover, the syringe was washed and primed with dichloromethane and methanol pre- and post-injection, and methanol solvent blanks were injected in between samples.

Stock solution containing $1 \mathrm{mg} / \mathrm{mL}$ CAF reference standard was prepared in methanol and stored at $-20^{\circ} \mathrm{C}$. Calibration curve points were obtained using the stock solution by incremental amounts between $10-500 \mu \mathrm{g} / \mathrm{mL}$ concentrations including $100 \mu \mathrm{g} / \mathrm{mL}$ of IS. Each calibration points were analyzed six times to evaluate the system precision and calibration curve was constructed through plotting peak area ratio against the ratio of CAF concentration to IS concentration.

Limit of detection (LOD) and limit of quantitation (LOQ) were estimated as $3.3 \mathrm{\sigma} / \mathrm{slope}$ and 10 $\sigma /$ slope of the calibration curve of CAF, respectively. As the lack of available certified reference material (CRM) of sport supplement for CAF; precision, accuracy and recovery studies were assessed by evaluating six replicates in three different concentrations $(50,100$ and $250 \mu \mathrm{g} / \mathrm{mL})$ of CAF spiked into caffeine-free powder samples. The satisfaction range was assumed as $80-120 \%$ for recovery, $15 \%$ bias for accuracy and $20 \mathrm{RSD} \%$ for precision. Additionally, in order to observe matrix effect, IS containing methanol solvent and caffeine free powder sample were analyzed and compared by overlapping chromatograms.

\section{Results and discussion}

\subsection{Performance Parameters of the Method}

According to the results, CAF and IS were found at retention time $8.87 \pm 0.01 \mathrm{~min}$ and $10.98 \pm$ $0.001 \mathrm{~min}$, respectively as shown in the chromatogram (Figure 1) obtained from a CAF containing sport supplement. There was no interfering peaks in chromatograms obtained from the matrices and extraction solvents. A linear regression of a five-point calibration curve of CAF including IS was used for quantification. System precision was determined by performing injection repeatability test $(n=6$ for each calibration points) and $\leq 9.0 \mathrm{RSD} \%$ values for $\mathrm{CAF}$ was found to be acceptable. The calibration curve for CAF was found to be linear in the range of $10-250 \mu \mathrm{g} / \mathrm{mL}$ with a correlation coefficient of 0.995 . The LOD and LOQ were calculated as $4.58 \mu \mathrm{g} / \mathrm{mL}$ and $13.74 \mu \mathrm{g} / \mathrm{mL}$, respectively.

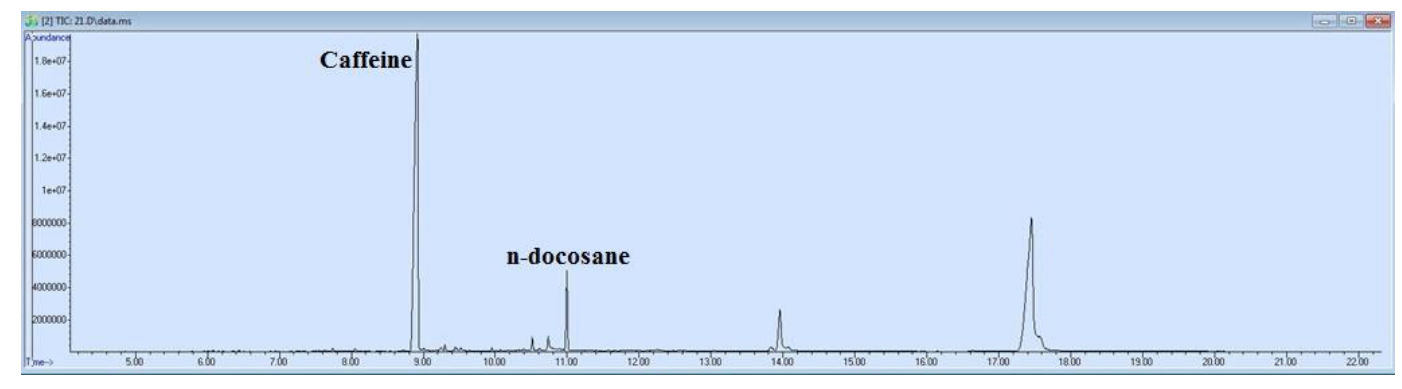

Figure 1. GC-MS chromatogram results of a sport supplement, containing caffeine and n-docosane (IS)

Accuracy, recovery and precision of the method was evaluated for caffeine in the spiked samples with three concentrations $(50,100,250 \mu \mathrm{g} / \mathrm{mL})$ which were shown in Table 1 . The bias correction values were found less than $4.93 \%$ also extraction efficiency was found satisfactory with $\geq 95.07 \%$ recovery with low RSD\% values $(\leq 2.40)$. 
Table 1. Performance parameters CAF determination in GC-MS method

\begin{tabular}{cccc}
\hline $\begin{array}{c}\text { Concentration } \\
(\boldsymbol{\mu g} / \mathbf{m L})\end{array}$ & $\begin{array}{c}\text { Average Recovery } \\
(\boldsymbol{\%})\end{array}$ & $\begin{array}{c}\text { Accuracy } \\
(\mathbf{B i a s} \boldsymbol{)})\end{array}$ & $\begin{array}{c}\text { RSD } \\
(\boldsymbol{\%})\end{array}$ \\
\hline $\mathbf{5 0}(\mathbf{n}=\mathbf{6})$ & 97.61 & 2.39 & 0.66 \\
$\mathbf{1 0 0}(\mathbf{n}=\mathbf{6})$ & 95.07 & 4.93 & 2.40 \\
$\mathbf{2 5 0}(\mathbf{n}=\mathbf{6})$ & 98.78 & 1.22 & 0.61 \\
\hline
\end{tabular}

\subsection{Analysis Results of Sport Supplements}

The GC-MS analysis of 41 sport supplements revealed that 17 (41.5\%) products were found to be contained caffeine, ranging from 4.52 to $471 \mathrm{mg} / \mathrm{mL}$. Labels were never mentioned caffeine existence in 3 (Product number 4, 8 and 9) of $17 \mathrm{CAF}$-containing products as indicated in Table 2. Besides, CAF amounts in contents were not stated in 3 of caffeine labelled products (Product number 3, 7 and 11). Eight of $17 \mathrm{CAF}-$ containing samples were found to be higher than stated on their labels (Product number: 1, 5, 6, 10,12,14, 16 and 17). All details of the aforementioned findings can be found in Table 2. The CAF amounts in $\mathrm{mg} /$ cup were calculated as considering the suggested amounts of caffeine in terms of cups (serving size) on each product, which indicates daily consumption (mg/day).

Table 2. Results of caffeine containing sport supplements and their label information $(n=17)$ Product

\begin{tabular}{llcccc}
\hline No & $\begin{array}{c}\text { Form of } \\
\text { Products }\end{array}$ & $\begin{array}{c}\text { CAF notification } \\
\text { on label }\end{array}$ & $\begin{array}{c}\text { Labeled CAF } \\
\text { amount }\end{array}$ & $\begin{array}{c}\text { Determined CAF } \\
\text { concentration }\end{array}$ & $\begin{array}{c}\text { Calculated CAF } \\
\text { amount }\end{array}$ \\
\hline & & & (mg/cup) & (mg/mL) & (mg/cup) \\
\hline 1 & Powder & Yes & 160.3 & 133.1 & 286.0 \\
2 & Powder & Yes & 100.2 & 34.7 & 104.4 \\
3 & Powder & Yes & unspecified & 364.2 & $521.7^{*}$ \\
4 & Powder & No & - & 25.0 & 30.0 \\
5 & Powder & Yes & 96.8 & 167.7 & 300.2 \\
6 & Tablet & Yes & 24.9 & 38.5 & 116.9 \\
7 & Powder & Yes & unspecified & 213.0 & $458.1^{*}$ \\
8 & Powder & No & - & 9.5 & 38.8 \\
9 & Powder & No & - & 253.0 & $455.4^{*}$ \\
10 & Powder & Yes & 225.4 & 172.3 & $550.6^{*}$ \\
11 & Powder & Yes & unspecified & 130.0 & 391.0 \\
12 & Powder & Yes & 145.1 & 73.4 & 220.2 \\
13 & Tablet & Yes & 50.0 & 4.6 & 18.1 \\
14 & Tablet & Yes & 24.9 & 197.3 & 236.4 \\
15 & Tablet & Yes & 200.1 & 30.3 & 182.2 \\
16 & Powder & Yes & 100.9 & 18.5 & 224.6 \\
17 & Powder & Yes & 150.6 & 471.5 & $546.4^{*}$ \\
\hline
\end{tabular}

*Refers to daily consumption amounts more than FDA recommendation

In total, 17 out of 41 products were found as caffeine-containing supplements, among which caffeine content of 3 were not labelled on products. In five of the investigated sport supplements, caffeine was found to be above the daily recommended intake level (highlighted as * in the Table 2) including the product number 9 which was not even labelled. Overall, according to Student t-test results significant differences were observed $(p<0,05)$ between the quantities indicated on the labels and the determined caffeine amounts in some products (number 1, 5, 6, 10, 12, 14 and 16). In response to this, CAF-containing products that stated only the presence of the substance (number 3, 7 and 11) were either 
at the recommended limit or above. In overall consideration of this study, the daily intake range of these products was found as 18.1-550 $\mathrm{mg} /$ day, while 8 of 17 caffeine detected products were found between 151-948\% higher than their stated label information with $361 \%$ mean value.

To compare our results with the literature, similar studies were investigated. A study reported by Andrews et al. in 2007 was observed that the caffeine amounts in 53 sport products ranged from 0.6 to $622 \mathrm{mg}$ /day. In addition, caffeine amounts in the investigated products were found up to $173 \%$ higher than labels claimed in the same study [17]. In another research conducted on solid and liquid capsules of dietary supplements, it was found that 6 of 105 liquid samples were above the daily limit of $400 \mathrm{mg}$ and approximately $20 \%(\mathrm{n}=97)$ of the analyzed solid capsules were contained more than claimed amount from 120 to $382 \%$ [1]. A different study from Brazil revealed that caffeine amounts in $\mathrm{mg} /$ day were determined in between $25 \pm 3.4$ and $1476.7 \pm 78.5$ for 94 supplements in the market; also, caffeine amounts were higher up to 524\% than labelled concentrations among analyzed products [21]. Neves et al. has also confirmed that capsules in the same package may contain different doses of caffeine and other byproducts due to insufficient standardization in these supplementary products. Additionally, even though the label claim does not change, it was found that the amount of caffeine in consecutive series of the same brand could considerably vary [1].

Considering aforementioned incompatibilities between labelled and measured amounts and safe daily intake limits of caffeine, consumption of these products may cause unpredictable serious health problems for user. Considering the frequency of sport training (twice or third times a week) and the amount of supplements consumed at a time, it can be estimated that the daily caffeine consumption limit ( $400 \mathrm{mg} /$ day) can be easily exceeded in a regular workout day. Therefore, possible side effects are easy to encounter. These effects mostly depend on consuming dose, condition of the trainer, type of exercise but are not limited only to these factors [3,4]. Besides, 5 of 41 products analyzed in this study were found above the daily safe limit solely; this means, without consuming any caffeine containing products such as coffee, tea or energy drinks, consumers of these products may already under serious health risks. As another clinically important observation is that 16 out of 17 caffeine containing products determined in this study were stated as pre-workout. It is known that all the exercises, without using any sport supplements, cause adrenergic stimulation effects such as increase blood pressure and heart rate [2123]. In the case of caffeine containing pre-workout product use, which stimulates the release of catecholamine, these adrenergic effects may increase dramatically. From the clinical aspect, excessive daily consumption of caffeine may increase health problems such as arterial hypertension, coronary heart disease, stroke, insulin insensitivity, and impaired glucose tolerance, as well as sleep problems, irritability, anxiety, headache, and restlessness [21-23]. In addition, if the regular user is under a treatment with medications they may interact with caffeine, which can lead to severe or fatal consequences. In a study, it was found that $200-300 \mathrm{mg}$ of caffeine daily intake increases the systolic blood pressure to $14 \mathrm{mmHg}$, diastolic blood pressure up to $13 \mathrm{mmHg}$ in normotensive individuals and these effects become clearer in hypertensive individuals [24]. As demonstrated by two separate metaanalyzes, people who consume more than 5 cups of coffee per day are at 40-60\% more risk of ischemic heart diseases (myocardial infarction and unstable angina) than non-consumers [24]. On the other hand, since the caffeine is a chemically addictive stimulant, withdrawal symptoms may be triggered in the case of caffeine consumption is reduced or cut. The severity of withdrawal symptoms may vary from individually, but generally include headache, anxiety, drowsiness, heart rhythm abnormalities, depression, muscle pain, nausea, vomiting, and lack of concentration [3,21,22].

On the growing concerns of the European Union countries regarding to the safety and quality of these supplements, new directives were introduced by the European Commission to protect public health. In particular, Regulation 2002/46/EC aims to protect consumers from potential health risks arising from these products and to avoid misleading information [25]. As for in our country, nutritional supplements, including sport supplements, are regulated Ministry of Agriculture and Forestry. FDA and EFSA, as worldwide official authorities, determined the maximum daily intake of caffeine as $400 \mathrm{mg}$ $[10,11]$. It is known that while the consumption of caffeine in low-to-medium doses $(3-6 \mathrm{mg} / \mathrm{kg})$ increases the performance in trained athletes, in high doses $(\geq 9 \mathrm{mg} / \mathrm{kg})$, to the contrary, it causes severe side effects and no contribution to performance at all [4]. 
As a result, it can be seen that on an ordinary training day, consuming more than the recommended dose or being under any treatment may cause severe adverse reactions, so consumers may be faced with serious health risks in their daily life. Hence, people who consume caffeine-containing sport supplements need to be more aware of the content of these products and possible side effects of caffeine since these supplements are sold on easily accessible platforms such as Internet, gyms, and pharmacies without any detailed advisory.

\section{Conclusions}

In this work, a simple and selective analytical method was successfully developed and validated for the detection and quantitation of caffeine in powder or tablet form of sport supplement products by GC-MS. The method was demonstrated to be highly reproducible, specific, precise, and accurate. To the best of our knowledge, there is no study in the literature determining the caffeine content in sport supplements available in Turkey market regarding to product's label claims and recommended daily intakes.

The inconsistency in labeling of these 41 sport supplements indicates the lack of quality control and inadequate supervision of sport supplement market. Due to the increased consumption of sport supplements in daily life, health professionals and authorities have important responsibilities to make sure the compliance of the labeling with the content in order to increase the safety in production, labeling and sale. Additionally, a separate notification should also be included in the label of caffeine containing products for possible adverse effects.

\section{Acknowledgement}

This research was supported by Research Fund of Istanbul University- Cerrahpasa with the project numbers 50936 and 20643.

\section{Supporting Information}

Supporting information accompanies this paper on http://www.acgpubs.org/journal/journal-ofchemical-metrology

\section{ORCID}

Eda Okuroglu: 0000-0002-4490-244X

Tugba Tekin: $\underline{0000-0001-6573-8426}$

Merve Kuloglu: 0000-0002-3670-3168

Selda Mercan: 0000-0002-0431-6972

Isil Bavunoglu: 0000-0001-5146-2254

Munevver Acikkol: 0000-0003-0642-6574

Zeynep Turkmen: 0000-0002-8250-7520

\section{References}

[1] D.B.J. Neves and E.D. Caldas (2017). Determination of caffeine and identification of undeclared substances in dietary supplements and caffeine dietary exposure assessment, Food Chem. Toxicol. 105, 194-202.

[2] S.R. Waldvogel (2003). Caffeine-a drug with a surprise. Angew. Chem. Int. Ed. 42(6), 604-605.

[3] B.F. Harland (2000). Caffeine and nutrition, Nutrition. 16(7), 522-526.

[4] E.R. Goldstein, T. Ziegenfuss, D. Kalman, R. Kreider, B. Campbell, C. Wilborn, L. Taylor, D. Willoughby, J. Stout, B.S. Graves, R. Wildman, J.L. Ivy, M. Spano, A.E. Smith and J. Antonio (2010). International society of sports nutrition position stand: caffeine and performance, J. Int. Soc. Sports Nut. 7(1), 5. https://doi.org/10.1186/1550-2783-7-5.

[5] M.A. Tarnopolsky (2008). Effect of caffeine on the neuromuscular system-potential as an ergogenic ai,. Appl. Physiol. Nutr. Metab. 33(6), 1284-1289. 
[6] M. Doherty and P.M. Smith (2005). Effects of caffeine ingestion on rating of perceived exertion during and after exercise: a meta-analysis, Scand. J. Med. Sci. Sports. 15(2), 69-78.

[7] B.B. Fredholm, K. Bättig, J. Holmén, A. Nehlig and E.E. Zvartau (1999). Actions of caffeine in the brain with special reference to factors that contribute to its widespread use, Pharmacol. Rev. 51(1), 83-133.

[8] M.S. Ganio, J.F. Klau, D.J. Casa, L.E. Armstrong and C.M. Maresh (2009). Effect of caffeine on sportspecific endurance performance: a systematic review, J. Strength. Cond. Res. 23(1), 315-24.

[9] A.B. Hodgson, R.K. Randell and A.E. Jeukendrup (2013). The metabolic and performance effects of caffeine compared to coffee during endurance exercise, PLoS One. 8(4), 1-10.

[10] EFSA. European Food Safety Authority, (2015). Scientific Opinion on the safety of caffeine, EFSA J. 13(5), 4102. Retrieved from: https://www.efsa.europa.eu/en/efsajournal/pub/4102.

[11] FDA Takes a Closer Look. Caffeine and Kids [Internet], (2018). [cited 2018 Dec 19]. Retrieved from: https://www.fda.gov/ForConsumers/ConsumerUpdates/ucm350570.htm.

[12] World Anti-Doping Agency. The world anti-doping code. The 2009 prohibited list international standard [Internet], (2009). [cited 2018 Dec 20]. Retrieved from: http://www.wada-ama.org.

[13] T.E. Graham (2001). Caffeine, coffee and ephedrine: impact on exercise performance and metabolism, Can. J. Appl. Physiol. 26(S1), S186-S191. https://doi.org/10.1139/h2001-046.

[14] S.D. Reid, J. Ramsarran, R.L. Brathwaite, S. Lyman, A. Baker, D.C. Cornish, S. Ganga, Z. Mohammed, A.T. Sookdeo and C.K. Thapelo (2015). Energy drink usage among university students in a Caribbean country: Patterns of use and adverse effects, J. Epidemiol. Glob. Health. 5(2), 103-116.

[15] D. Wikoff, B.T. Welsh, R. Henderson, G.P. Brorby, J. Britt, E. Myers, J. Goldberger, H.R. Lieberman, C. O'Brien, J. Peck, M. Tenenbein, C. Weaver, S. Harvey, J. Urban and C. Doepker (2017). Systematic review of the potential adverse effects of caffeine consumption in healthy adults, pregnant women, adolescents, and children, Food Chem. Toxicol. 109, 585-648.

[16] S.M.F. Bessada, R.C. Alves and M.B.P. Oliveira (2018). Caffeine-based food supplements and beverages: Trends of consumption for performance purposes and safety concerns, Food Res. Int. 109, 310-319.

[17] K.W. Andrews, A. Schweitzer, C. Zhao, J.M. Holden, J.M. Roseland, M. Brandt, J.T. Dwyer, M.F. Picciano, L.G. Saldanha, K.D. Fisher, E. Yetley, J.M. Betz and L. Douglass (2007). The caffeine contents of dietary supplements commonly purchased in the US: analysis of 53 products with caffeine-containing ingredients, Anal. Bioanal. Chem. 389(1), 231-239.

[18] D.M. Bordin, B.B. Bettim, G.C. Perdona, E.G. De Campos and B.S. De Martinis (2017). Understanding alterations on blood and biochemical parameters in athletes that use dietary supplements, steroids and illicit drugs, Toxicology. 376, 75-82.

[19] H.J. Kim, J.H. Lee, H.J. Park, S.H. Cho, S. Cho and W.S. Kim (2014). Monitoring of 29 weight loss compounds in foods and dietary supplements by LC-MS/MS, Food Addit. Contam. Part A. 31(5), 777 783.

[20] C. Oellig, J. Schunck and W. Schwack (2018). Determination of caffeine, theobromine and theophylline in Mate beer and Mate soft drinks by high-performance thin-layer chromatography, J. Chromatogr. A, 1533, 208-212.

[21] C. Viana, G.M. Zemolin, L.S. Müller, T.R. Dal Molin, H. Seiffert, L.M. de Carvalho (2016). Liquid chromatographic determination of caffeine and adrenergic stimulants in food supplements sold in Brazilian e-commerce for weight loss and physical fitness, Food Addit. Contam. Part A. 33(1), 1-9.

[22] G.B. Keijzers, B.E. De Galan, C.J. Tack and P. Smits (2002). Caffeine can decrease insulin sensitivity in humans. Diabetes Care. 25(2), 364-369.

[23] P. Nawrot, S. Jordan, J. Eastwood, J. Rotstein, A. Hugenholtz and M. Feeley (2003). Effects of caffeine on human health, Food Addit. Contam. Part A. 20(1), 1-30.

[24] J.V. Higdon and B. Frei (2006). Coffee and health: a review of recent human research, Crit Rev. Food Sci. Nutr. 46(2), 101-123.

[25] A. Petroczi, G. Taylor and D.P. Naughton (2011). Mission impossible? Regulatory and enforcement issues to ensure safety of dietary supplements. Food Chem. Toxicol. 49(2), 393-402.

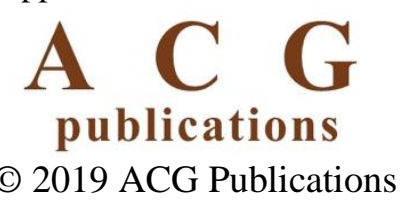

